\title{
Gastric banding to treat obesity: band-aid or breakthrough?
}

\author{
Kenneth S Polonsky and Samuel Klein
}

The worldwide epidemic of obesity is associated with an increase in serious medical complications that substantially raises morbidity risks and health care costs. This situation is aggravated by the fact that most current therapies for obesity have limited efficacy. Lifestyle interventions to reduce caloric intake and increase physical activity can work but multidisciplinary teams are often needed to ensure that the necessary changes are made and maintained. Moreover, even these interventions have high relapse rates. Drug therapies for obesity have been disappointing; some have been associated with serious side effects and withdrawn from the market, and those currently approved provide only $8-10 \%$ weight loss within 1 year, with gradual regain thereafter (Klein $\mathrm{S}$ et al. [2002] Gastroenterology 123: 882-932).

Bariatric surgery is currently becoming the most effective and successful way to achieve weight loss. Various techniques may be used; Roux-en-Y gastric bypass and laparoscopic adjustable gastric banding (LAGB) are the most commonly performed bariatric procedures worldwide. LAGB involves laparoscopic placement of a band around the most proximal region of the stomach. A subcutaneous access port is used to introduce saline into a balloon on the inner surface of the band; expansion of this balloon restricts the gastric luminal diameter. The band circumference can be adjusted according to the patient's clinical response. This technique is less invasive than other bariatric surgical procedures and does not change intestinal anatomy. Nonetheless, LAGB can be associated with severe complications, including death, in a small percentage of patients.

Two randomized, controlled trials compared the weight-loss efficacy of LAGB with that of aggressive, nonsurgical therapy (O'Brien PE et al. [2006] Ann Intern Med 144: 625-633 and
Bariatic surgery

is currently

becoming the

most effective

and successful

way to achieve

weight loss

KS Polonsky is the

Adolphus Busch

Professor and

Chairman of the

Department of

Medicine, Washington

University School

of Medicine, and

$S$ Klein is the

William H Danforth

Professor of Medicine

and Nutritional

Science and Director

of the Center for

Human Nutrition at

Washington University

School of Medicine in

St Louis, MO, USA.

\section{Competing interests}

The authors have declared associations with the

following companies: Amylin

Pharmaceuticals, Dannon-

Yakult, EnteroMedics,

Ethicon Endo-Surgery,

GlaxoSmithKline, Merck

Laboratories, Metabolex

Sanofi-Aventis and Solae

Company. See the article

online for full details of these

relationships.

www.nature.com/clinicalpractice doi:10.1038/ncpendmet0889
Dixon JB et al. [2008] JAMA 299: 316-323). In one trial, individuals with class I obesity (BMI $30-35 \mathrm{~kg} / \mathrm{m}^{2}$ ) were treated with LAGB or a verylow-calorie diet, pharmacotherapy and behavioral therapy. At 2 years, the nonsurgically treated patients had lost $5 \%$ of their initial weight, corresponding to $21.8 \%$ of their excess weight (defined as weight above that corresponding to a BMI of $25 \mathrm{~kg} / \mathrm{m}^{2}$ ) and the LAGB group had lost $21.6 \%$ of their initial weight, which equaled $87.2 \%$ of their excess weight. In the second study, obese patients who had type 2 diabetes and class I or II obesity (BMI $30-40 \mathrm{~kg} / \mathrm{m}^{2}$ ) were randomly assigned treatment with either LAGB or conventional diabetes therapy, which included lifestyle modification (diet and exercise). At 2 years, diabetes remission was achieved in $73 \%$ of those treated with LAGB but in only $13 \%$ of those given conventional treatment. In addition, reductions in blood glucose are often seen within the first few weeks of LAGB and other bariatric procedures, even before substantial weight loss has occurred. Thus, novel hormonal and biochemical mechanisms might play a part in the improvement of metabolic outcomes that occurs following bariatric surgery.

Studies of LAGB are needed to address several important questions. First, what is the long-term safety of the procedure? Second, do weight loss and diabetes remission persist beyond several years? Third, what are the optimum upper and lower BMI thresholds for treatment with LAGB in obese patients with and without type 2 diabetes? Fourth, does LAGB have a role in treatment of severely obese children? Finally, what are the mechanisms responsible for decreased energy intake and improvement in glucose homeostasis? The answers to these questions will determine the place for LAGB in the long-term management of obesity and its complications. 